\title{
Remote Laser Laboratory: Lifebuoy for Laser Engineering Curriculum
}

\author{
A Safe and Cost-Effective Solution for Hazardous Lab Environment \\ http://dx.doi.org/10.3991/ijoe.v8i2.1761 \\ Igor E. Titov ${ }^{1,2}$, Olga A. Smirnova ${ }^{2}$, Alexander N. Glotov ${ }^{2}$ and Alexander D. Golovin ${ }^{2}$ \\ ${ }^{1}$ EPFL, Lausanne, Switzerland \\ ${ }^{2}$ Bauman Moscow State Technical University (BMSTU), Moscow, Russia
}

\begin{abstract}
Laboratory experience represents an essential part of the engineering curriculum and even more so for in the area of laser engineering. However, gaining such practical experience may be hazardous for both the students and expensive equipment. This paper presents a ready-to-use solution that can be used for both e-learning and safe remote operation: Remote Laser Laboratory (RLL). Software and hardware solutions are presented in this work. In addition, a short description of ongoing student activities within the RLL framework is given.
\end{abstract}

Index Terms-e-learning, expensive equipment, hazardous environment, labicom, laser, remote lab.

\section{INTRODUCTION}

One might find the engineering, educational and scientific areas in which a remote mode of operation is not just desirable or cost-effective, but also the only possible way to teacher medium and large-sized groups of students. Remote laser laboratories can not only substantially increase the number of students who can be served, but also provide several additional advantages. For example, laser equipment can pose an immediate threat to an unskilled operator because there are a variety of scenarios which can lead to permanent damage of vision or complete blindness. This, in itself leads to a considerable loss of actual contact time using the equipment, not concentrating on engineering and physical questions of studied or measured phenomena, while going over rather extensive safety procedures. Even if instructed thoroughly, there is still no guarantee that all safety measures will be obeyed in all cases. Statistically, the greater the number of students using a laboratory, the greater the chance of a laboratory accident occurring. Online laboratories contribute to cost effectiveness and overall safety.

Unskilled operators also pose a threat to expensive equipment. Because educational and research laboratories have limited budgets to acquire often expensive equipment and maintenance, a major malfunction of any part of equipment might cause long-term or permanent suspension of laboratory activities that can undermine the entire curriculum or experiment schedule. Also, minor malfunctions caused by unskilled lab users might lead to long downtimes. For example, just touching the movable elements on a laser bench may lead to complete readjustment of almost all optical parts. Furthermore, large numbers of students require more room and supporting infrastructure.
Larger-sized groups working in a lab is not compatible with a clean environment and reproducible results. The remote mode of operation eliminates all of the aforementioned cons of traditional laboratories. In such a mode, it is possible to maintain equipment in a clean environment cared for by highly a skilled technical staff without direct exposure of unskilled lab users to hazardous and/or expensive equipment. Combining such benefits with those of e-learning pedagogy (flexible lab and student schedule, global presence, resource exchange with educational partners, etc.) it is evident that for hazardous and/or expensive laboratories, the remote mode of operation is the only possible way to deliver rich lab experiences to a greater number of students. Studying lasers as an engineering subject requires students participate in extensive theoretical and practical activities. Laser laboratories pose a threat to human eyesight if not used properly; they use dangerous voltages to pass energy to the power supply unit and photoelements, they employ easily breakable and expensive optical elements, they require dustless air conditions and a vibrationless optical bench. On the other hand, all Remote Laser Laboratory (RLL) parameters can be controlled in a very safe and reliable manner. A laser bench with optical elements can be enclosed in a sufficiently clean glass box using air filtering and higher pressure, thus isolating it from the surrounding environment. Also, an optical bench for an experimental laser setup can be mounted on a special stabilizing platform to eliminate vibrations and misalignment. These measures lead to a high quality of mirror alignment and the reproducibility of experiment results and measurements.

The benefits over local mode make RLL a perfect candidate for switching on remote operation. The local laser engineering laboratory at Bauman Moscow State Technical University (BMSTU) was transformed into a remote lab project and a beta version of RLL [1] is presented in this paper.

\section{RLL HARDWARE}

\section{A. Quantron}

Quantron provides both a gain medium and a cooling cavity in one mechanical structure. RLL uses solid-state active element on YAG:ND ${ }^{3+}$ (yttrium aluminum garnet with neodymim). The cooling cavity is filled with water and is connected to the water tank. The system uses forced water cooling while the power supply unit is turned on. 


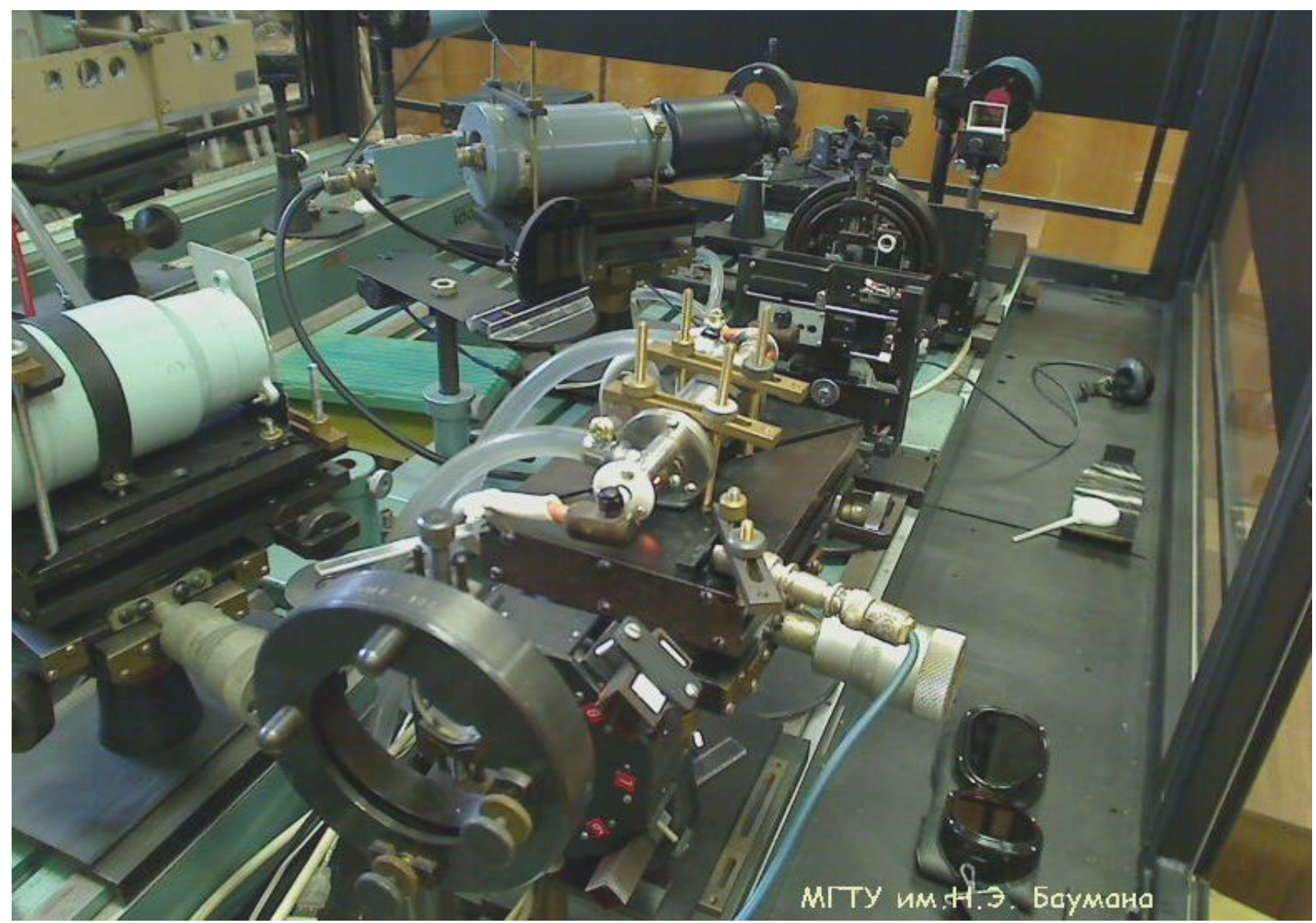

Figure 1. Web-camera view of RLL physical equipment on optical laser bench

\section{B. Web-cameras}

RLL can use up to five web-cameras but currently is using only two. Other camera places are reserved for future use. RLL uses commercial USB web cameras with a resolution of $800 \times 600$ pixels. A view from one of the cameras is presented on Figure 1. Currently, images are available in MJPEG, forcing the web browser to rewrite old images with a sufficient but not guaranteed rate. This causes pauses in the videos when the internet bandwidth is low or the CPU is heavily loaded. There are a few possible solutions under consideration to avoid this problem. One of them is to use compressed streaming video, thus easing requirements for the internet channel and client computer. Also, this will take an advantage of hardware graphics acceleration where possible. Consequently, this measure will also increase the recording rate at the server allowing to show the flash of light during laser shots, thus augmenting the students' perception of reality by means of visualization. Presently, such a flash of light during discharge can be seen through MJPEG, but this is not always guaranteed.

\section{Control Unit}

The RLL control unit interacts with the optical functional elements (Q-switch, diaphragm, non-linear crystal, etc.) and communicates with a computer through the USB interface. At the driver level, the RLL software utilizes the VISA standard for communication with the control unit.

\section{Power Supply Unit}

The power supply unit (PSU) controls the pumping of energy, discharge frequency and pilot arch. It also initiates discharge events (and therefore, the laser shot). It can produce discharges only if cooling system is operational.

\section{E. Oscilloscope}

The RLL uses a USB oscilloscope that conforms to VISA drivers. It has $200 \mathrm{MHz}$ of bandwidth and a $1 \mathrm{GS} / \mathrm{s}$ sampling rate, which allows it to register signals up to 2 ns in width.

\section{F. Joulemeter}

The energy measurement unit (joulemeter) incorporates a photoelectric cathode, capacitance integrator, ADC and microcontroller. Measured energy is transmitted to the RLL lab server with the help of WinUSB driver.

\section{G. Step-motors and actuators}

Step-motors and actuators control different functional parts of RLL instrumentation, i.e. Q-switchers, mirrors, optical filters, diaphragm diameter and non-linear crystal. Each of these elements is a custom-made electromechanical structure controlled by the RLL Control Unit. 


\section{RLL SOFTWARE}

Initially, the software part of the RLL was realized only in NI LabView in hope that users would install the LabView run-time engine and use Remote Panels to access the laboratory. But such a solution has proven to be very impractical, both from the point of view of user unfriendliness and of programming difficulties encountered during the RLL software test period. There also were other issues discussed in [article on delivering labview software to clients in browser]. To overcome many obstacles of NI Remote Panels, new client-server architecture was conceived. This suggests using greatly renewed (and even redone) the legacy RLL program written in LabView with a new client program written in Adobe Action Script (for Flash) to communicate with each other through TCP/IP. New RLL network topology conforms to the Labicom topology and meets its remote lab topology requirements. [2]

\section{A. Client-side software}

The RLL web-server is hosted by Labicom. In this way potential users have 24/7 access to schedule, learn and access other essential information,even if the RLL labserver is turned off. For this reason, we do not have to deal with so many networking, security, financial, administrative and programming issues connected with hosting professional web-server.

The client-side part of RLL is a rich internet application in Flash (fig. 2).

This technology has many strong points for remote laboratory applications, in particular for RLL.

1) Maximum coverage of users. Flash applications (SWF-files) are supported by all modern webbrowsers on all platforms. It means that RLL is really accessible from any computer in the world, be it on Windows, Mac or Unix/Linux operating systems. The Adobe player plugin is installed on more that 99\% of all internet-enabled browsers [3], making it an absolute leader in its ability to be used across platforms and its and deliverability.

2) Information security. Flash technology implies a safe unintrusive environment on the user's computer since all potentially dangerous operations cannot be performed by running flash software at all or may be performed only after the user's explicit consent.

3) Effective usage of internet traffic. SWF Flash file is a compressed version of source code, meaning that it is optimized for Internet since the Flash virtual machine is already presented in the browser.

4) Thin client paradigm. The resulting client-side application is a 'thin client', i.e. there is efficient division of labor between server (that controls equipment and organizes data transmission) and client (that shows control elements to user and displays data).

5) Rich internet application. Flash technology allows persons to deliver very eye-catching and customizable user interfaces. Modern users are accustomed to professional design and expect to see appealing images on the screen.

\section{B. Server-side software}

Sever-side RLL is written in the NI LabView graphical programming language. It is great in communication with

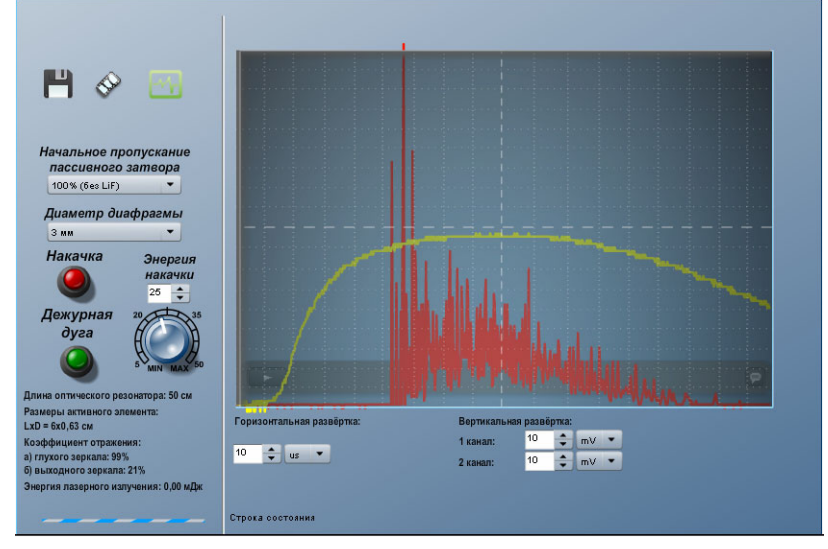

Figure 2. RLL client graphical user interface

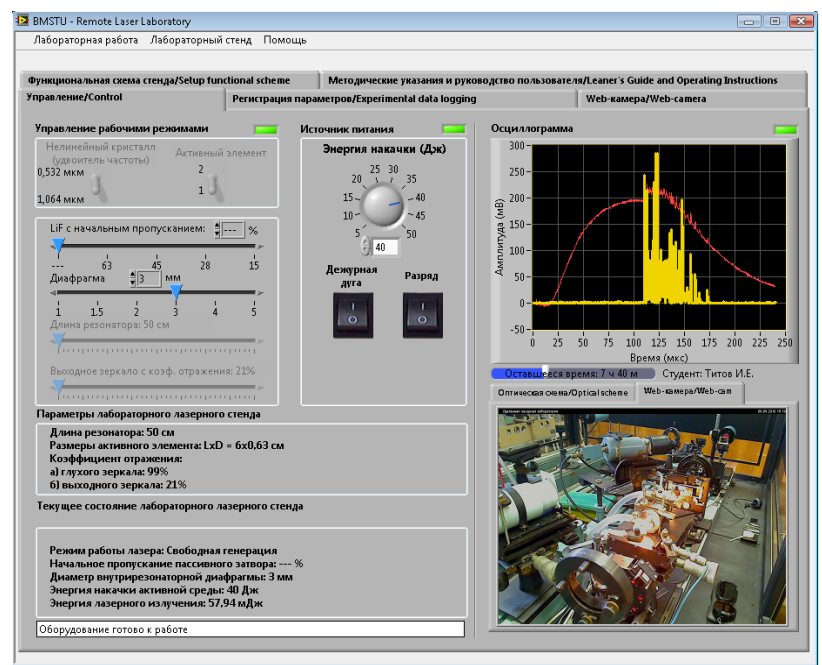

Figure 3. RLL lab-server main tab

different devices and it is also gaining momentum as a general purpose programming language. This fact allows the implementation of a great deal of logic on the server side. Its object-oriented capabilities are also used throughout the algorithm. The Graphic user interface of the RLL lab server consists of tabs (fig. 3). Each of these tabs focuses on some particular aspect of RLL operation: advanced control capabilities, web-cameras settings, RLL general settings, current optical scheme on the laser bench, etc.

- The Control algorithm. The RLL server is fully responsible for all direct communication with lab instruments. It uses parallel loops to take advantage of a multicore processor. It also has a system of interlocks preventing simultaneous operation of potentially conflicting equipment. For example, it makes it impossible to produce a laser shot while the Q-switch or non-linear crystal is moving, thus preventing equipment from being damaged.

- Laboratory administrator interface. The RLL administrator is a key part of the whole system: it maintains the physical equipment, determines laboratory working hours, creates and manages instructor accounts. It also determines the global availability of equipment and instruments on the laser bench.

- Alarm system. It is essential to have a fast response to arising hardware malfunctions since in remote mode there is a queue of students waiting for their 
PAPER

REMOTE LASER LABORATORY: LIFEBUOY FOR LASER ENGINEERING CURRICULUM

timeslots. Downtime can be effectively minimized if the lab administrator knows immediately about the problem. To this end, The RLL is equipped with a sound alarm system that activates if some equipment fails to operate. Also, the lab administrator receives an e-mail with a description of the problem.

- Logging system. Every user action is stored in the log-file. It allows persons to know the sequence of actions of each lab user. It is useful in programming and pedagogical ends and also can help in preventing abuse and misuse of the system.

- TCP/IP server. The RLL server communicates with RLL clients through TCP sockets. It waits for incoming connections and when a new request for a connection is received, the lab server demands authentication information. If this information corresponds to user's time slot for the current moment, the lab server establishes a permanent connection through the TCP socket. Otherwise, a request for connection is rejected. Connection terminates when the current lab session expires.

\section{Information Security questions}

Information security is invisible to RLL users but presents one of the main challenges during the RLL development process. On the client side, security is provided by using 'sandbox', which is safe environment in which Flash applications operate. On the server side there are different layers of security: hardware level (system of interlocks), access layer (only known users can gain a control over real equipment), logging layer (all users' actions are monitored, analyzed and stored). And between the RLL lab server and the RLL client, there is an extensive layer of transmitted data protection: TCP packets are encrypted with AES 256 (Advanced Encryption Standard 256-bit key) algorithm. AES 256 is a very robust symmetric key algorithm that has a good performance (high speed and low RAM requirements).

\section{Test and time reservation system}

As to test and time reservation, RLL fully relies on Labicom services. A student is not eligible to have an access to real equipment unless he or she has reserved a free time slot in the RLL schedule before this time slot has expired. To do so, a student has to successfully pass the entry test. Both activities are done on Labicom website [2].

\section{RLL USAGE By STUDENTS}

After having passed an entry test and reserved a time slot, a student is allowed to be connected to real equipment (at this specific time slot). By default, one lab session takes thirty minutes, but it can be changed by the professor. During this time, students can disconnect from the RLL and connect to it again. After his or her time has expired, the lab session will be terminated automatically, giving the place to next student. Depending on what settings a professor imposes on any given lab session or student, it is possible to conduct different experiments on the same equipment. One can measure energy, time and spectral or spatial characteristics of laser radiation which is too much for a single lab session from the point of view of material digestion.
Let us present a road map of student activities in RLL while participating in investigating energy characteristics of a solid-state laser. At first, the student assembles the optical scheme for the first part of the work (free-running generation), i.e. he or she chooses the exit mirror, active medium and a diaphragm. All needed measuring devices and optical filters turn on automatically when the student changes something on the functional scheme. Then the student pumps energy and detects a threshold of generation with the help of energy measuring devices and a digital oscilloscope. After that he/she can find a functional dependence of output energy on pumping energy within specified limits. The RLL lab-server saves all the oscillograms and characteristics on its hard drive and, in the end, sends the file to the student's e-mail. With these oscillograms he can make a rough estimate of pump duration, total generated pulse width, delay time between pump front and output pulses and amplitude-time structure of those pulses. After that, the student has to make a graph of the dependency of output energy on pumping energy. The second part of this laboratory is a giant pulse mode investigation. Students fulfill the same sequence of operations. They assemble scheme adding a Q-switching optical shutter. The design of a Q-switcher is to maintain up to seven Q-switches with different initial beam transmissions. Once again, students gradually change the pumping energy, detect the threshold of generation and afterward use pump energy slightly over the threshold. For every lithium fluorine used as passive Q-switches the students must record the pulse shape and width. After measurements, students can plot the dependency of pulse width and output energy on a ratio of initial to threshold population.

In such activities students perform the same operations and can see and detect the same results as if they were performing these lab activities in the laser engineering laboratory, situated in the educational institution. This clearly shows many of the benefits of remote operation compared to the traditional benefits of on-site operation of real equipment.

\section{CONCLUSIONS}

Remote Laser Laboratories combines the strong points of a complex engineering laboratory with absolute physical safety of lab users who work behind their displays at home. This adds up to numerous benefits provided by elearning method of instruction. From now on, laser engineering curriculum will no longer suffer from the lack of individual laboratory experience when lab work can be performed by groups of students, or even demonstrated to students by an instructor. RLL is evolving in its due course: there are many new features under active development. In the next version of RLL we are planning to have new energy measurement unit, control unit and CCD-camera.

\section{ACKNOWLEDGMENT}

We would like to thank professor Victor Petrov for great teaching methodology help who kindly provided us with a professor's standpoint on remote educational product features along with some more specific advice on quantum electronics and laser engineering questions. 
PAPER

REMOTE LASER LABORATORY: LIFEBUOY FOR LASER ENGINEERING CURRICULUM

\section{REFERENCES}

[1] Igor Titov, Olga Smirnova, Alexander Glotov, Alexander Golovin "Remote Laser Laboratory: First Demonstration. Worldwide substitution for laser engineering lab", Remote and Virtual Labs '11 conference proceedings, in press, 2011.

[2] http://www.labicom.net/labs/rll, Remote Laser Laboratory at Bauman Moscow State Technical University (Russia).

[3] http://www.adobe.com/products/player_census/flashplayer/, "Content played back in Flash Player reaches 99\% of Internet viewers"

\section{AUTHORS}

Igor Titov currently has double affiliation with École Polytechnique Fédérale de Lausanne (CH-1015 Lausanne, EPFL, Switzerland) and with Bauman Moscow State Technical University (105005, Moscow, 2nd Bauman- skaya st., 5, BMSTU, Russia). He leads software development of Remote Laser Laboratory at BMSTU. (e-mail: igor.ev.titov@gmail.com).

Olga Smirnova is an electronics and laser engineering professor at BMSTU. She is a head of RLL project. (e-mail:osmirnova@rocketmail.com).

Alexander Glotov is a professor of digital circuits and digital electronics at BMSTU. He leads hardware development of RLL. (e-mail: aglotov@mail.ru).

Alexander Golovin is a professor of quantum electronics and laser engineering at BMSTU. He is a first user of RLL among professors. (e-mail: golovin@bmstu.ru).

Received 23 July 2011. Published as submitted by the authors 27 April 2012. 